\title{
Belphégor
}

\section{Le Vice Chic}

Faits divers et représentations des bas-fonds à la Belle Époque brésilienne

\section{Valéria Guimarães}

\section{(2) OpenEdition}

1 Journals

\section{Electronic version}

URL: http://journals.openedition.org/belphegor/733

DOI: 10.4000/belphegor.733

ISSN: 1499-7185

\section{Publisher}

LPCM

\section{Electronic reference}

Valéria Guimarães, «Le Vice Chic », Belphégor [Online], 14 | 2016, Online since 23 October 2016, connection on 19 April 2019. URL : http://journals.openedition.org/belphegor/733 ; DOI : 10.4000/ belphegor.733

This text was automatically generated on 19 April 2019

\section{(c)}

Belphégor est mis à disposition selon les termes de la Licence Creative Commons Attribution - Pas d'Utilisation Commerciale - Pas de Modification 4.0 International. 


\title{
Le Vice Chic
}

\author{
Faits divers et représentations des bas-fonds à la Belle Époque \\ brésilienne
}

Valéria Guimarães

1 Correspondant à ce qu'on a appelé «l'âge d'or de la presse » (Smythe 2003; Charle 2004, p. 136), le passage du XIXe au XXe siècle a connu une diffusion sans précédent des idées, unissant les continents par le biais des imprimés. Malgré son implantation tardive, la presse brésilienne de la fin du XIX a rejoint ce circuit d'informations aux circulations intenses, favorisé par l'utilisation commerciale du télégraphe.

2 L'arrivée quotidienne des télégrammes avec leurs nouvelles toutes fraîches, alliée à la traditionnelle importation de journaux étrangers, dynamise le marché de la presse, tout en mettant en place de nouveaux modèles de journalisme. C'est pendant cette période que le sensationnalisme atteint son premier pic au Brésil. Deux matrices étrangères sont à l'œuvre - l'une est américaine, l'autre est française. Malgré un décalage perceptible dans le temps, c'est le modèle français de la presse à sensation qui semble davantage avoir captivé le lecteur brésilien, avec son ton ouvertement littéraire et sociologique qui entend fouiller la nature humaine, à la recherche des raisons qui mèneraient à la " dégénérescence morale ", pour utiliser une expression en vogue dans le discours « hygiéniste».

3 Marlyse Meyer (1996) a bien exploré la réception des feuilletons français au Brésil ; la publication des faits divers dans les journaux brésiliens répond à une logique similaire, mais sans la synchronie des feuilletons publiés presque simultanément en Europe et au Brésil. Il est possible de trouver dans la presse brésilienne du début $\mathrm{du} \mathrm{XX}^{\mathrm{e}}$ siècle des références qui renvoient à un imaginaire partagé. La circulation accrue des informations l'autorise, soit par l'entremise de nouvelles technologies comme le télégraphe, soit par la propagation physique des supports de l'imprimé.

4 Dans cet article, nous nous concentrerons sur les représentations des bas-fonds dans les journaux brésiliens publiés au cœur de l'axe éditorial formé par les plus grandes villes brésiliennes de l'époque, Rio de Janeiro et São Paulo. Ces périodiques circulaient partout dans le pays et servaient à la fois de références en termes journalistiques et d'agents de diffusion du nationalisme républicain. Notre choix se justifie ainsi par le rôle joué par ces 
journaux dans le contexte de diffusion de la culture lettrée au Brésil, un pays dans lequel l'analphabétisme était la règle et dont la réalité ne correspondait pas à celle de la modernité européenne.

5 Dans ce contexte, la publication des faits divers par les journaux a eu une importance considérable dans la diffusion de valeurs morales, même en tant que contre-exemple. Elle a aussi facilité la réception de l'imprimé au sein de couches sociales qu'il n'avait pas touchées auparavant. Ici, l'exploitation de la dimension orale du récit s'est appuyée sur des structures et des thèmes bien enracinés dans l'imaginaire. Pour expliquer le succès qu'ont connu les faits divers, il faut envisager la façon dont ils formulent et diffusent des stigmates sociaux (en nous éloignant de la perspective qui les considère uniquement comme des productions aliénantes), mais aussi comprendre leurs autres fonctions sociales. Ils offrent en effet des modèles de comportements autant que des références topographiques utiles aux vagues incessantes d'immigrants qui arrivent dans une ville en constante mutation (Kalifa 1995).

Les faits divers obtiennent une place croissante dans les quotidiens brésiliens. Le Jornal do Comércio apparaît en la matière comme une honorable exception, face à la production périodique brésilienne du début $\mathrm{du} \mathrm{XX}^{\mathrm{e}}$ siècle, qui réserve des sections spéciales aux nouvelles scandaleuses. Nous avons choisi le thème des bas-fonds car, dans les sources étudiées, les affaires qui sont en rapport avec le milieu de la drogue et de la prostitution à São Paulo et à Rio de Janeiro apparaissent pleines de références à l'imaginaire français des bas-fonds, lequel y est représenté de façon particulièrement séduisante, amorçant un dialogue riche avec la littérature européenne. Ce ne sont donc pas uniquement les formats, mais aussi les imaginaires sociaux qu'ils véhiculent, qui sont les objets de circulations transatlantiques.

\section{Le club de la mort}

7 Le 12 mars 1917, le journal de São Paulo A Gazeta publie un dessin de Voltolino ${ }^{1}$. Celui-ci nous donne quelques indices des images des bas-fonds qui circulaient à l'époque :

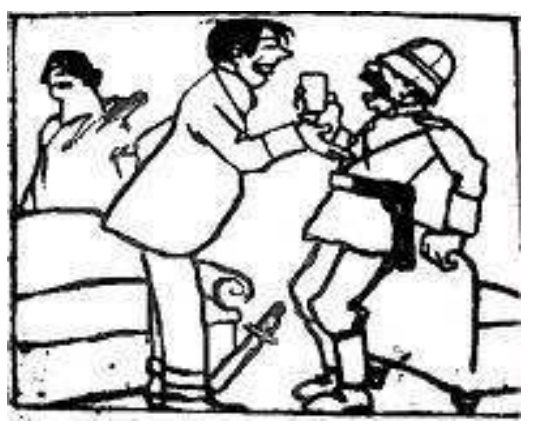

Figure 1 : Le Vice Chic : encore une noble campagne contre la cocainne dévastatrice par Voltolino² (A Gazeta, 12/03/1917)

8 Ce dessin était inclus dans la rubrique A Semana Gaiata (Figure 2), publiée tous les lundis, dans laquelle le dessinateur humoristique commentait les faits divers de la semaine précédente publiés par le journal.

9 Voltolino est l'héritier d'une longue tradition brésilienne de l'illustration humoristique, mais il semble aussi connaître la production étrangère, comme "Les Gaîtés de la Semaine » dessinées par Draner pour Le Supplément Illustré du Petit Journal et « La Semaine 
Illustrée » de Henriot, publiée dans Le Petit Journal. Il apparaît donc comme un passeur culturel entre les deux pays.

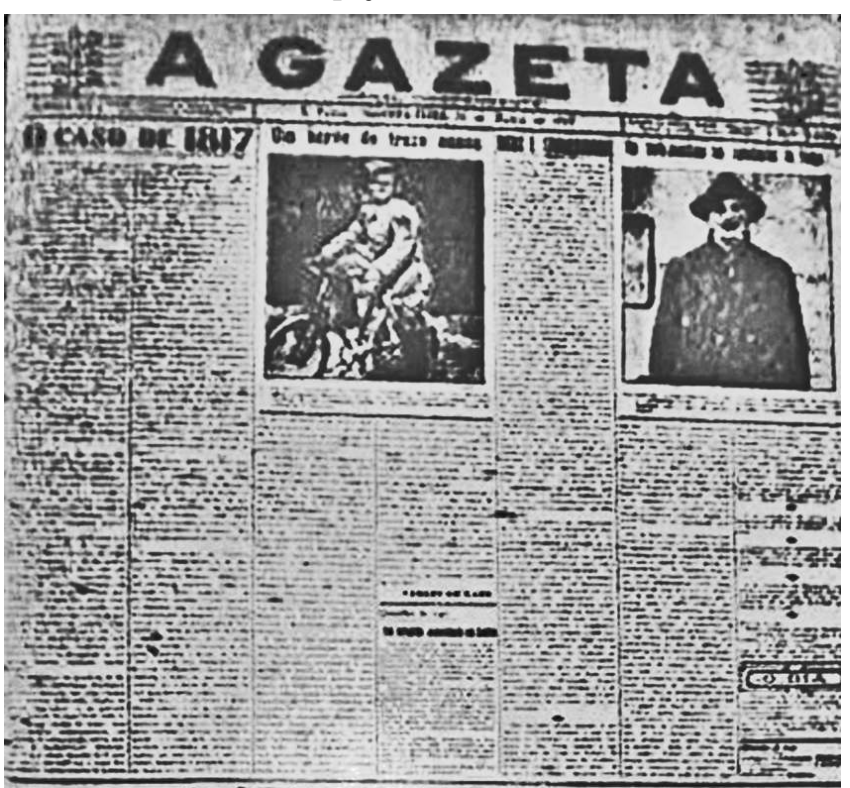

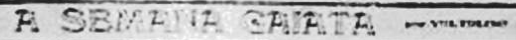

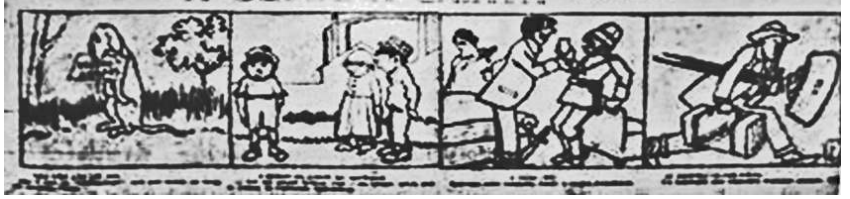

Figure 2 : Dans le « rez-de-chaussée » de la première page, tous les lundis, A Semana Gaiata par Voltolino (A Gazeta, 12/03/1917)

10 Voici la rubrique de Draner, Les Gaîtés de la Semaine (Figure 3), qui semble être la référence de Voltolino pour l'A Semana Gaiata: 


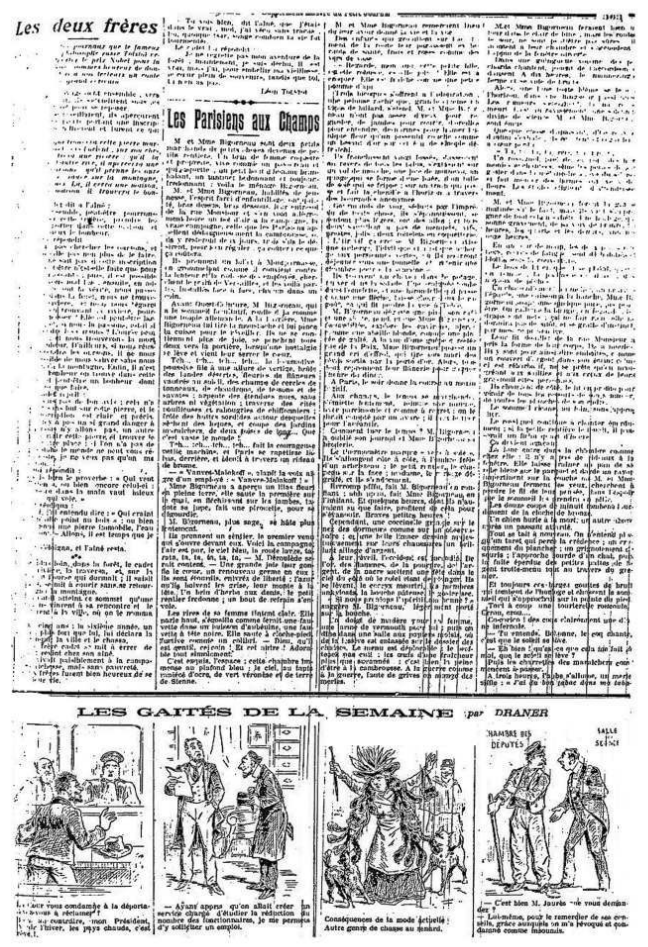

Figure 3 : Les Gaîtés de la Semaine par Draner (Supplément Illustré du Petit Journal, 12/11/1910)

11 La similarité entre les deux rubriques est grande, soit dans leur format, soit dans la proposition de commenter les événements de la semaine. Nous observons ci-dessous la rubrique du français Henriot, dans La Semaine Illustrée (Figure 4), qui sert aussi 
d'inspiration à Voltolino dans les années 20 lorsqu'il illustre la rubrique A Semana à Lápis (Figure 5) :

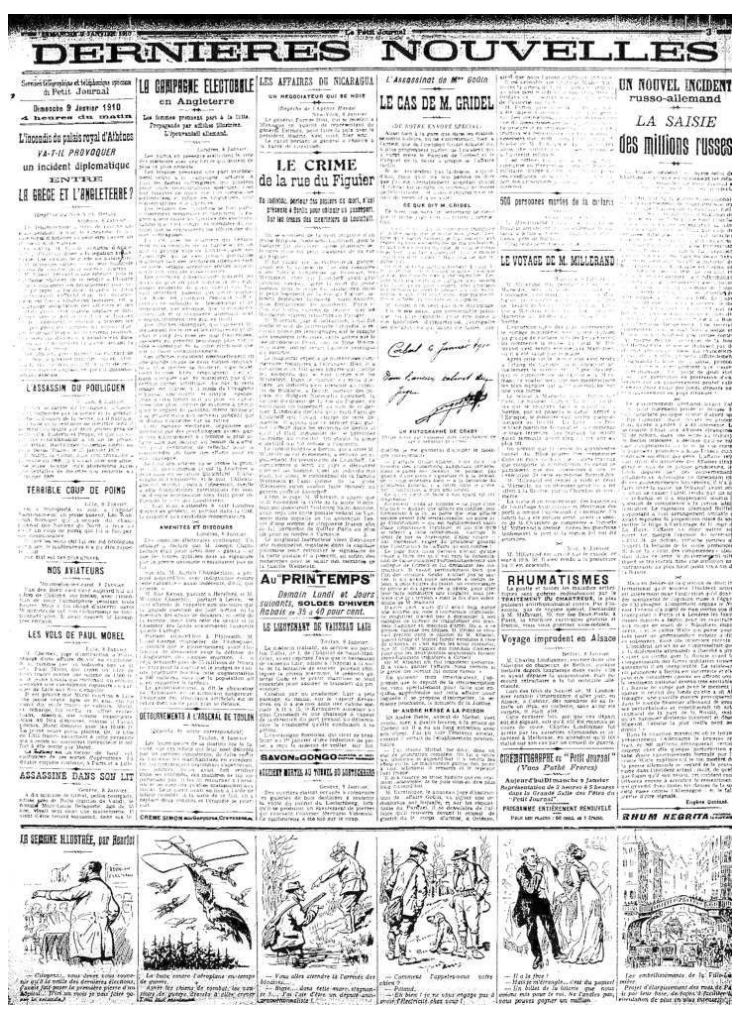

Figure 4 : La Semaine Illustrée par Henriot (Le Petit Journal, 09/01/1910)

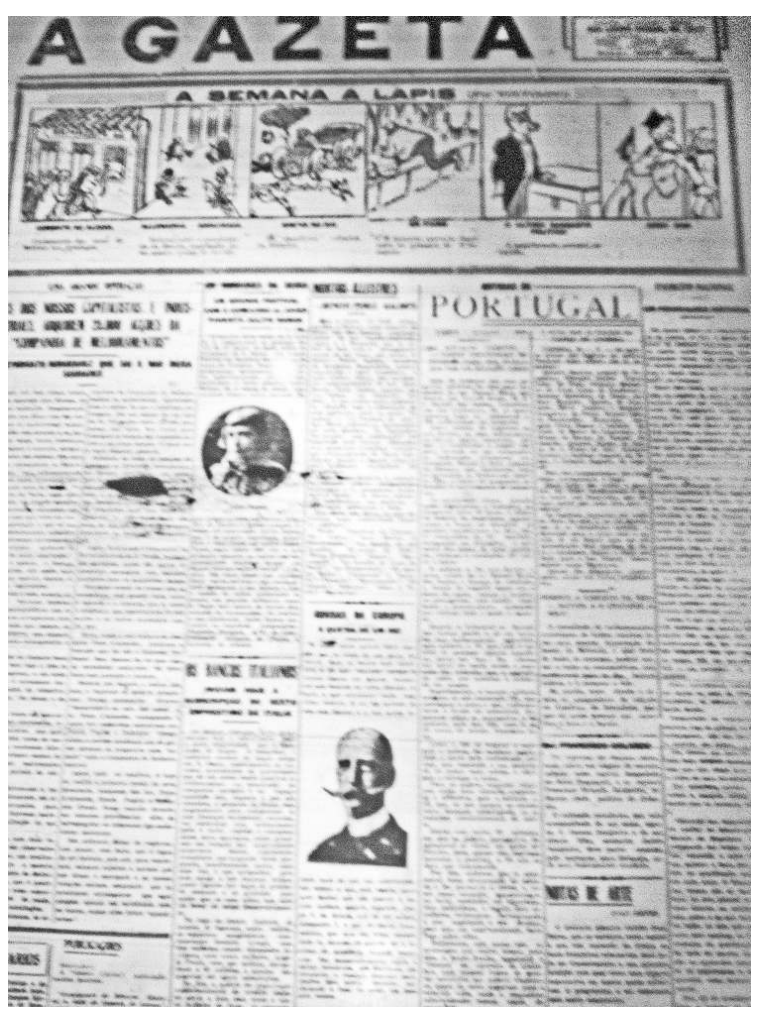

Figure 5 : A Semana à Lápis par Voltolino (A Gazeta, 05/01/1920) 

quotidien de São Paulo comme thèmes dans lesquels des types brésiliens apparaissent dans une perspective assez caractéristique de son genre d'humour, avec des traits simples et peu de texte.

En ce que concerne l'analyse de la Figure 01 , on observe sur le dessin de la troisième vignette de la séquence, au premier plan, un jeune homme qui convainc le policier d'échanger une matraque contre un verre. Les vêtements du personnage signalent sa richesse, et son attitude témoigne de sa volonté de corrompre le policier. L'espace diégétique renvoie à l'univers des garçonnières, que louaient de jeunes brésiliens à São Paulo, et dans lesquelles se réunissaient amis et petites amies. Au second plan, la figure féminine peut aussi incarner une prostituée cherchant à échapper à la police. On ne peut pas comprendre le dessin sans son titre, «Le Vice Chic - Encore une noble campagne contre la cocaïne dévastatrice ", mais celui-ci n'est pas suffisant pour que la scène soit complètement déchiffrée.

En effet, ce titre joue sur une inversion par rapport à la scène : s'il s'agit bien d'une campagne contre les drogues, l'action du policier semble anodine face à la capacité de douce résistance des consommateurs. Pour comprendre les représentations qui apparaissent ici, il faut lire les nouvelles auxquelles Voltolino fait référence. Ses dessins dialoguent avec la rubrique «Última Hora » du même quotidien, laquelle a coutume de publier des faits divers sensationnels. La campagne contre les drogues à laquelle le dessinateur se réfère a comme titre « O Club da Morte » (« Le club de la Mort »).

en 1906 par Adolpho de Campos Araujo, le journal A Gazeta apparaît dans un contexte d'intense concurrence. Il se peut que cela soit la raison pour laquelle ses dirigeants en ont fait un organe " du soir » (qui parait l'après-midi), tourné vers le divertissement, avec moins d'analyse politique ou économique que les titres matinaux. En voulant se montrer " moderne ", le journal a fait le choix d'une mise en page tapageuse, avec beaucoup d'illustrations, de rubriques, de gros titres. Les campagnes publiques dans lesquelles il s'engage entretiennent la proximité avec son lectorat, politiquement sousreprésenté, et éloigné des exiguës politiques publiques. En fait, la Gazeta joue un rôle de « représentante du peuple». Et cela semble avoir été une des principales caractéristiques de ce journal, le plus sensationnaliste de São Paulo à cette époque.

, après la mort de son fondateur, il est devenu propriété exclusive de l'avocat João Dente, qui a fait en sorte que les pages du journal deviennent une véritable extension des tribunaux. On ne dispose pas des données officielles sur les tirages, mais le quotidien luimême annonce qu'il était lu par plus de quinze mille personnes (A Gazeta, 12/11/1916). La rubrique " Última Hora », de son côté, apparaît en 1915 et remplace "Scenas de Sangue ", s'alignant sur la vogue de rubriques aux titres similaires, consacrées à mettre le lecteur au courant des dernières nouvelles et dépêches - comme son titre, littéralement «la dernière heure ", l'indique - et qui existaient dans plusieurs autres journaux brésiliens ( Gazeta de Notícias, Correio da Manhã et Diário Popular) et français (« Dernière Nouvelles » - Le Journal ; « Dernière Heure » - Le Petit Parisien).

17 Il y avait plusieurs organes de presse qui menaient des campagnes «d'assainissement moral » ou « d'hygiène sociale ». Le Jornal do Brasil et la Gazeta de Notícias se sont engagés contre le «chamanisme» et $O$ Estado de S. Paulo contre la prostitution, par exemple. Pourtant, c'était la Gazeta de São Paulo qui semblait mettre l'accent sur ce journalisme dit " citoyen ", même si d'autres journaux lui accordent également une place significative. 
On trouve ainsi des campagnes en faveur du désarmement et contre la possession illégale d'armes à feu (A Gazeta, 26/06/1915) ; contre les employés de la compagnie de distribution de gaz opérant des relevés suspects des compteurs (A Gazeta, 05/07/1915); contre le «médecin Don Juan » qui a séduit sa cliente mariée (A Gazeta, 26/10/1916) ; contre les maisons de passe et le proxénétisme (Gazeta de Notícias, 05/05/1910; Gazeta de Notícias, 30/01/1915; A Gazeta, 28/10/1916) ; contre la consommation et la vente de drogues, reprise par Voltolino (12/03/1917); pour la décence dans la presse (A Gazeta, 17/03/1917) ; contre la hausse des prix des négociants (A Gazeta, 20/05/1917) ; contre l'action du gouvernement en réaction à la grippe espagnole (A Gazeta, 22/10/1918); contre les chauffeurs assassins (A Gazeta, 09/01/1920); contre les "dandies", des hommes qui harcèlent des jeunes filles de bonne famille dans le Triangle ${ }^{3}$ (A Gazeta, 23/02/1920) et beaucoup d'autres.

Ces campagnes oscillaient, comme nous avons pu le remarquer, entre le combat contre la marginalité et la représentation des souffrances du travailleur face à la hausse des prix. Cette relation ambiguë essayait d'opposer la figure du travailleur honnête à ceux dont le comportement ne coïncidait pas avec l'éthique productive, ce qui révélait l'ambiance tendue qui a dominé la Première République (1889-1930) et qui a connu son apogée en 1917 à São Paulo, en raison de la crise que s'y était installée de façon dramatique. Quelques mois après la publication de la rubrique de Voltolino, l'oppression des travailleurs allait engendrer la plus grande grève pauliste (voire brésilienne), la Grève Général de 1917. Les journées de juillet, d'inspiration anarchiste, en pleine Première Guerre, ont paralysé et terrorisé les classes aisées ${ }^{4}$.

L'intention de criminaliser les ouvriers au chômage, qui vaquent sans occupation et sont souvent les meneurs des grèves, domine les représentations de telles campagnes. Même s'ils ont surtout été nombreux pendant le $\mathrm{XX}^{\mathrm{e}}$ siècle - au moment de la Grande Immigration dans certaines régions du Brésil, notamment São Paulo dans les années de 1890 - ces types de récit avaient commencé à circuler dès la fin du XIX siècle dans plusieurs journaux, comme par exemple « Cáftens Portugueses - Tudo n'esta terra é assim mesmo !... » («Proxénètes Portugais - Tout sur cette terre est comme ça! » Gazeta de Notícias, 23/01/1880). Dans ce texte, le journaliste se plaint de la police qui, malgré le fait d'avoir expulsé « une bonne douzaine de ces trafiquants, juifs, russes et allemands », était toujours tolérante avec les proxénètes :

Proxénètes Portugais - tout sur cette terre est comme ça

Et jusqu'à présent on s'en est accommodé !

Pourtant, d'autres proxénètes, de nationalité portugaise, sont restés ici, et font venir, d'eux même ou par l'intermédiaire de leurs amantes, de pauvres jeunes filles qui sont tenues dans les maisons closes de façon à rester éternellement sous le joug de la prostitution et qui leur offrent des gains journaliers fabuleux.

Ces proxénètes portugais se rient des autorités car ils possèdent l'appui de gros protecteurs qui se croient à la tête de ce pays.

Les chefs de ces proxénètes, hommes et femmes, ont leurs noms à la police, ils sont connus par le $3^{\text {e }}$ Chef de Police et sont dénoncés dans des articles de la Gazeta de Notícias.

En avant donc, M. le Chef de Police, et délivrez cette ville de tels monstres comme vous nous avec délivré des juifs.

La justice n'a pas de privilège, elle doit être la même pour tous.

À suivre.

La voix de Dieu. (Gazeta de Notícias, 23/01/1880) 


\section{que l'urbanisation contribuait à cet accroissement :} policiers, tout en indiquant de quelle manière se constitue un réseau lucratif de connivence avec le crime. Le fait que la presse souligne l'intégrité du chef de la police de São Paulo démontre à quel point la corruption était bien implantée. Dans le dessin de Voltolino, la représentation de la relation entre les bas-fonds du crime et la police corrompue n'est que la reprise d'un imaginaire déjà bien sédimenté en 1917. Indépendamment de la réalité du crime, ce qui nous intéresse, de fait, est la façon dont les représentations des bas-fonds dans les dessins de Voltolino et dans les faits divers mettent en scène un imaginaire récurrent dans lequel les personnages ne sont pas seulement des prostituées et leurs maquereaux, un trafiquant et des drogués, mais également la police et les " gros protecteurs " personnifiant la corruption des autorités. La perception du trafic de drogues et de la prostitution montre une police assez différente de celle qui apparaît comme restauratrice de l'ordre dans la plupart des faits divers. Les bas-fonds, face obscure de la société, tombent comme un voile noir sur tous ceux qui transitent dans son orbite.

On peut affirmer que les bas-fonds fonctionnent comme une construction, "une collection d'idées sur le crime médiatisée par l'appareil culturel et juridique " (Shore 2007, p. 43) que l'élite plaque sur les classes ouvrières. Heather Shore indique que les représentations des bas-fonds liées à l'idée de constitution d'un monde à part deviennent cohérentes dès le XVIII ${ }^{e}$ siècle. Elles sont présentes dans la culture populaire, mais aussi dans le discours de journalistes, de policiers et d'hommes politiques (Shore 2007, p. 43). De son côté, Dominique Kalifa (2013) affirme que les bas-fonds « désignent à la fois des lieux, des individus et des comportements » (2013, p. 26). Ils peuvent être interprétés, dans l'expérience de la ville en crise, comme un type d'« urbanophobie » (2013, p. 26). São Paulo était sûrement ce type de ville funeste, effrayante, une vraie capitale des bas-fonds "que les tensions sociales agitent et que les pulsions de son imaginaire enflamment " (2013, p. 27). La différence est que, à l'époque de notre étude, le début du XXe siècle, si les rénovations d'inspirations haussmanniennes sont déjà expérimentées à São Paulo, elle ne peuvent répondre aux effets de l'augmentation de la population en raison de l'immigration. L'attitude prédatrice de l'élite foncière, acharnée dans la défense de ses intérêts, contribue aussi à l'échec des efforts de transformation de la capitale paulista.

Le contexte est donc favorable à l'essor d'un imaginaire des bas-fonds et de leurs «mystères", à São Paulo autant qu'à Buenos Aires ou à Rio. Au Brésil, l'usage de 
représentations liées à ce que l'on appelle les bas-fonds se diffuse au début du siècle de façon plus étendue et gagne de l'écho dans les quotidiens. Dès la fin du XIX ${ }^{e}$ siècle, les journaux commencent à identifier certains groupes à la criminalité. Les groupes de « capoeiras » en sont un exemple. Métis, noirs et au chômage, ils sont associés à d'autres marginaux comme les pickpockets, les voleurs de bijoux, les falsificateurs de billets, parmi d'autres délinquants qui se réunissent en bandes pour mieux agir. Un processus identique mêle les prostituées et le monde de la drogue, dont la puissance d'évocation est liée à sa dissémination accrue.

Les places publiques, les marchés et les zones de grande concentration apparaissent comme les lieux du crime par excellence. Ce sont des espaces ouverts, comme la rue où l'on doit rester vigilant. Cependant, les espaces fermés ne sont pas exempts de tragédies : des bars à mauvaise réputation, mais aussi les pensions, les chambres d'hôtel et les garçonnières sont les décors de scènes de sang et de trafic de drogue. La représentation des bas-fonds sert alors à donner une matérialité au monde du crime de façon simple et stéréotypée, et les autorités juridiques autant que les médias mettent en circulation pour le grand public ces formes condensées qui permettent de caractériser un certain genre de criminalité.

Le journal remplit toujours son rôle de dénonciateur des failles des pouvoirs publics et des institutions dans leurs réponses aux demandes en termes d'urbanisation. Il ne s'agit pas de s'opposer frontalement au gouvernement, mais de faire entendre la voix du « bon citoyen». D'autre part, la presse s'engage du côté du pouvoir dans de bruyantes campagnes hygiénistes comme celle contre les maisons closes et le proxénétisme publiée dans la rubrique "As Campanhas de Saneamento Social» (Les campagnes d'assainissement social) dans le journal A Gazeta. Ce rôle normatif de la presse prend la forme d'un discours eugéniste, composante des orientations sanitaires dominantes dans les villes qui connaissant une urbanisation rapide.

\begin{abstract}
Les Campagnes d'Assainissement Social
Prostitution et Proxénétisme

Un autre domaine qui nous a orienté dans cette campagne est l'incitation à la défense de la santé publique contre le danger des maladies infectieuses et chroniques que la prostitution apporte avec elle et qui, dans le régime dans lequel nous vivons, constituent des facteurs colossaux de dégénérescence de notre race. [...] Les pensions de haut et de bas niveau ne sont pas soumises à des visites médicales. Les prostituées sont des foyers impunis et silencieux de la propagation non seulement de maladies chroniques, mais également de tous les maux vénériens qui sapent l'organisme et affaiblissent le caractère (A Gazeta, 28/10/1916).
\end{abstract}

Tout en exposant une immense photo des maisons de prostituées de la rue São João accompagnée du sous-titre « Alguns antros da rua de S. João » [ "Quelques antres de la rue São João »], cette campagne est également en rapport avec la série de reportages dans la rubrique « Última Hora » du même journal A Gazeta appelée « O Club da Morte » « Última Hora ", avec laquelle le dessin de Voltolino dialogue, et qui concerne le monde de la drogue, et surtout la consommation de cocaïne.

Depuis mars 1916, le journal faisait de gros efforts contre la propagation de cette drogue. Cette initiative se trouve étroitement liée aux campagnes contre la prostitution :

Le club de la Mort

La cocaïne détruit $\mathrm{S}$. Paulo

Visions de délire - le commerce clandestin - les "pensions chics» - les vendeurs à la sauvette

La « cocaïne-manie » est le mal qui détruit actuellement, de façon étonnante, la 
capitale, enveloppant dans son tourbillon destructeur la jeunesse qui s'effondre et se détruit dans les délires du terrible toxique, s'enivrant dans les cabarets et pensions qui pullulent tels des antres de perdition.

La vente de la coca, ou la poudre, comme on dit en argot, se fait sans scrupules par des moyens variés ; il y a même une classe d'individus qui se consacre uniquement à ce rentable et ignoble commerce.

L'étonnement est encore plus grand lorsque l'on apprend que, comme s'il s'agissait d'un sport, d'un divertissement licite, il y a un club organisé pour l'offre, la pratique et la jouissance de la cocaïne : l'étonnant Club de la Mort !

Demain nous commencerons la publication de notre sensationnel reportage sur ce thème. utilisait la cannabis indica pour ses vertus thérapeutiques. Elle soignait la «difficulté à respirer, les ronflements, les flatulences, l'aspiration sibilante [...] et la toux ", en plus de l'asthme et de l'insomnie (Fonseca 1988, p. 239). Elle était acceptée socialement, et la publicité pour les paquets de cigarettes était faite dans les journaux, les magazines et les almanachs brésiliens. Diffusée dès le XVIII ${ }^{\mathrm{e}}$ siècle, elle a été introduite au Brésil par les Portugais qui la cultivaient pour l'utilisation de la fibre de chanvre dans les cordes utilisées par la marine. Les esclaves la consommaient en cigarettes, suivant une habitude probablement apportée d'Afrique, et l'utilisation du "pango » - on l'appelait ainsi - a commencé à être combattue, parce qu'elle mobilisait la matière utilisée pour la confection des cordes des navires. Le laboratoire français Grimault \& Cie. était le principal fabricant des cigarettes indiennes, mais il était aussi possible de trouver une version nationale chez les herboristes de São Paulo (Fonseca 1988, pp. 235-238). Dans les années vingt, la vente commence à en être contrôlée et la loi impose que sa délivrance soit motivée par un avis médical.

L'autre drogue populaire à l'époque est l'opium, limitée par la loi à l'utilisation médicale. Dans les années 1910, sa consommation récréative devient à la mode à Rio de Janeiro. Bientôt, l'opium est introduit à São Paulo (Fonseca 1988, p. 241) et atteint à son apogée dix ans après, déclenchant une vague "d'opiomanie ». La cocaïne reste cependant la drogue la plus courante. «Les premiers contacts de la police avec la substance apparaissent dans des cas de suicides, de tentatives de suicide ou d'accidents causés par un usage excessif, chez des malades cherchant à soulager des douleurs » (Fonseca 1988, p. 245).

L'utilisation de ces substances s'est diffusée alors que progressait le style parisien de finesse et d'élégance art déco en architecture et dans les illustrations, dans les vêtements, le design de voitures et la littérature : « Parmi ces voluptés, il y avait un appétit pour l'utilisation de drogues qui exaltaient les sens, ouvraient les chemins vers des nouveaux plaisirs, rendaient leurs adeptes plus intelligents, plus sensibles et surtout plus modernes » (Resende 2006, p. 18). 

Londres, de New York ou de Buenos Aires, de São Paulo ou de Rio de Janeiro. Il est impossible d'estimer à quel point ce lien est le fruit d'une construction, tirant force et structure de la littérature et de la presse. Les drogues participaient d'un imaginaire social chic et civilisé, auréolé de modernité. Il faut rappeler que le trafic de cocaïne était international, la France et l'Europe étant, en général, perçues comme les plus grands centres de consommation et de propagation. Et « même les productions bizarres de l'art moderne » étaient associées à sa consommation (Sevcenko 1992 [1998, p. 85]).

Naturellement, le contexte agit sur l'attention plus ou moins grande accordée à ces questions, comme l'indique Dominique Kalifa, et aussi bien São Paulo que Rio de Janeiro faisaient partie du circuit de trafic de drogues, qui entretenait une forme de «francisation » des habitudes. Cet imaginaire se nourrit enfin de l'excitation qui envahit les âmes dans cette confluence de changements technologiques significatifs d'une part et d'autre part du pouvoir de destruction de la Grande Guerre. Entre la vision apocalyptique de la fin du monde et le rêve d'une nouvelle société (Sevcenko1992 [1998, p. 168]), surgit cette agitation généralisée dont profitent les moyens de communication, tout en construisant des images effrayantes.

Cependant, le texte journalistique privilégie moins le portrait fidèle de la réalité qu'une "identification dramatique » du lecteur avec l'écart, par le biais d'un "récit pseudolittéraire " (Motta 2002, p. 314). Le portrait des bas-fonds que font les journaux ne correspondait pas nécessairement aux bas-fonds réels, qui devaient être plus sales et malsains que les décors exotiques et idéalisés décrits par les journalistes. Les faits divers offrent des informations dont la valeur réside dans le pouvoir d'être stimulant, ce qui dépend justement de l'imagination du journaliste. La caricature ou le dessin humoristique qui accompagnent le texte, le rendent plus lisible pour un public élargi; la série de nouvelles accompagnées des dessins de Voltolino a plus de chance de toucher les lecteurs - c'est-à-dire, d'augmenter les ventes de la Gazeta.

Les principaux traits et représentations des bas-fonds étant définis, nous pouvons revenir $\mathrm{au}$ "Club da Morte ». Cette désignation a été donnée par la presse à un groupe de jeunes gens riches de São Paulo qui consommaient des drogues pour se divertir. À partir des années 1910, on commence à mettre en cause la cocaïne dans des décès de jeunes issus des couches aisées. Ces cas préoccupent l'élite sociale, d'autant plus qu'on suppose l'existence de relations avec des figures des bas-fonds. Ces affaires impliquaient des personnes liées au monde des cabarets, comme les actrices, qui, souvent, étaient également des prostituées. Ainsi, en 1912, le suicide d'une actrice française («Loucura Fatal » [La Folie Fatale], O Estado de S. Paulo, 04/02/1912) ${ }^{5}$ est l'occasion implicite, pour la presse, d'associer bohême, dégénérescence et prostitution. La jeune femme serait morte après avoir consommé de l'éther, de la morphine, de la cocaïne et de l'opium. Dans l'imaginaire collectif, la prostituée de luxe était associée à l'Europe et à Paris, mais appartenait toujours à la pègre.

Lorsque les victimes du "Club da Morte " étaient des jeunes de la haute société, le traitement médiatique se révélait différent. Pour préserver leur famille, on ne citait pas leur nom, par exemple.

DERNIERE MINUTE

Le Club de la Mort

Les cas récents de suicide de jeunes appartenant à des familles respectées de São Paulo, et qui sont la conséquence de l'excitation provoquée par le terrible toxique,

Belphégor, 14 | 2016 
sont la preuve évidente que la cocaïne est en train de détruire S.Paulo.

LES ANTRES

On a pénétré dans un de ces antres atroces, lequel, d'ailleurs, s'est présenté à nous avec tous les ornements envoûtants d'un appartement chic, rayonnant de lumière, décoré avec un art raffiné, exhalant des arômes subtils qui aiguisent les sens.

Ici et là, des divans couleur abricot clair ou bleu serein en riche soie sur lesquels sont couchées, dans des poses voluptueuses, des femmes presque nues, en maillot, qui rient bruyamment au milieu de phrases plaintives tout en vidant, l'un après l'autre, des verres d'un capiteux champagne.

C'est une orgie des sens dans l'ivresse de la volupté et de l'alcool. Avec des airs de gentleman, habillés sagement, se détachent les silhouettes des adolescents... (A Gazeta, 09/03/1917).

On y identifie quelques éléments présents dans le dessin de Voltolino : le jeune homme riche, bien habillé. Les divans de l'appartement, destinés aux membres du club et aux femmes, probablement des prostituées. Le décor est décrit comme un lieu « d'orgie des sens ", dégageant une ambiance fascinante, chic, avec un art raffiné, mais plus proche d'un paysage de Baudelaire que d'un antre brésilien.

Le 10 mars, l'article est plus long et apporte plus de détails :

\section{Dernière Minute}

Le Club de la Mort

La cocaïne détruit S. Paulo

Le commerce clandestin - les " pensions chic » - les vendeurs à la sauvette

La Police doit agir

Ce qui est sûr c'est que, dans cette capitale, un grand nombre d'individus névropathes, pour mieux satisfaire leurs jouissances, ont fondé, avec toutes les dispositions, un club dont le seul but est d'administrer à ses associés l'usage du terrible alcaloïde. Effrayant !

[...] Peu avant, ils étaient heureux, satisfaits, pleins de joie, et juste après ils étaient dans une chambre, couchés par terre, avec le crâne traversé par une balle! Dehors, dans le vertige destructeur de la coca, il reste toujours un membre du Club de la Mort. [...] En outre, tous les membres du fatidique Club de la Mort sont des personnes qui appartiennent à notre plus noble société. Si on ne donne pas leurs noms, c'est simplement par respect pour leurs familles, lesquelles, d'ailleurs, n'ont que très peu ou aucune responsabilité dans les dérèglements de quelques-uns de leurs membres. LES VENDEURS

Sans scrupules, avec d'énormes moyens, comme s'ils voulaient répandre la mort sur toute une ville, ils sont partout.

[...] Et, dans une des pensions chic traîne ce terrible trafiquant qui exploite la vie et la santé d'autrui, en retirant de ce commerce honteux l'argent avec lequel il fait ses figurations habituelles dans des limousines luxueuses ou au bras de certaines demimondaines.

Un pharmacien, qui ne connaît rien en pharmacologie, profite de sa position pour disposer, dans une pharmacie, d'une grande quantité de cocaïne qu'il répand, pour un prix exorbitant, dans les maisons closes.

QUELQUES « CHAUFFEURS »

Ce sont d'autres misérables qui ne se contentent pas d'escroquer les clients des taxis , mais qui s'adonnent également au commerce interdit de la poudre. Beaucoup de ceux qui se garent à la place Paissandu apportent avec eux des flacons de cocaïne qu'ils vendent au prix de 5,8 , et même 10000 réis. [...]

LA NUIT DU « COCAÏNISTE »

On n'imagine pas la fièvre avec laquelle le drogué attend la tombée de la nuit, moment qu'il préfère pour s'adonner à sa terrible passion. Il est aisé pour lui de trouver la coca. Dans certaines pensions, elle est entre les mains de plusieurs femmes dont nous avons les noms dans notre cahier. Pour les proies qu'elles 
séduisent, elles ont toujours à portée de main le toxique destructeur pour garantir une vie de luxe facile pour elles-mêmes et leurs gigolots (sic) ou les maquerois (sic) [maquereaux].

Il devient indispensable que la police, par une action énergique, agisse contre ceux qui poussent leurs pauvres victimes au suicide. [...]

Il y a eu, d'après ce que l'on suppose, une certaine complaisance pour les maîtres de la cocaïne. [...] Aujourd'hui nous imprimons la reproduction photographique de trois flacons que nous avons obtenus avec beaucoup de facilité auprès de trafiquants de cocaïne.

Nous avons bon espoir que nos efforts contre le funeste vice qui dégrade la jeunesse paulista seront couronnés de succès (A Gazeta, 10/03/1917).

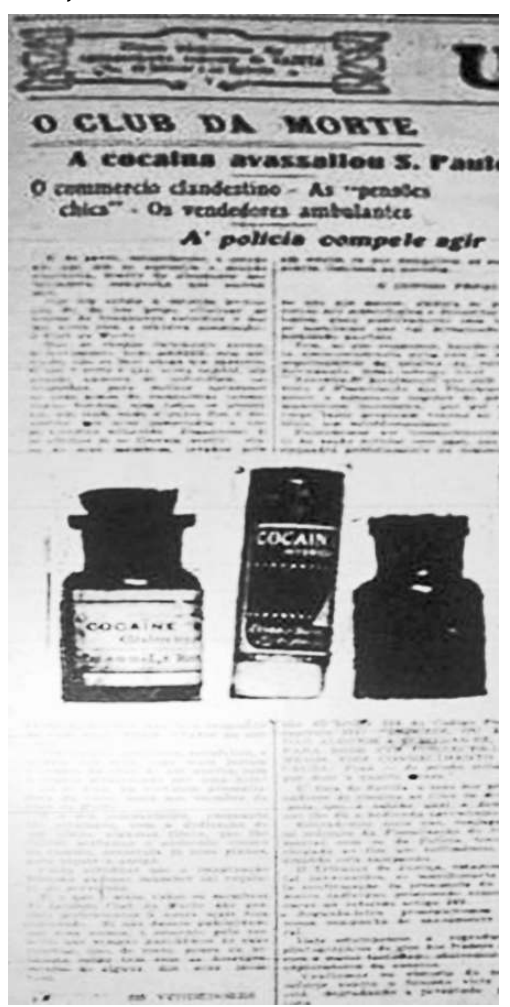

Figure 6 : Le Club da Mort La cocaïne détruit São Paulo6 (A Gazeta, 10/03/1917) premier cercle de personnages des bas-fonds; les trafiquants, de demi-mondaines, les pharmaciens, les chauffeurs et les proxénètes les accompagnent, et forment ensemble une escadrille du crime agissant contre la bonne société.

S'agissant des vendeurs, l'édition du 12 mars avertit :

LES VENDEURS

Les vendeurs à la sauvette sont multiples: des "chauffeurs", des garçons, des portiers, des employés des bars, des simples particuliers et même... des personnes bien placées font le vil commerce de la cocaïne, s'enrichissant grâce à la démoralisation et à la « debacle » (sic) des malheureuses victimes de cet alcaloïde. ( A Gazeta, 12/03/1916)

40 Le journaliste indique les endroits où a lieu le trafic:la "Pension Negrinha", la «Pharmacie de la rue Consolação », les « maisons closes de la rue Ipiranga », la « maison de la rue Conselheiro Crispiniano » (A Gazeta, 12/03/1916).

DANS UNE PENSION

Très connue et fréquentée par des célébrités du monde politique, on y voit 
fréquemment d'horribles « cocaïnomanes » dans des scènes de dépravation, comme celle qui a eu lieu, il y a quelques jours, dans une petite salle où l'on respirait des essences merveilleuses et où un luxe oriental était le décor admirable d'épisodes tristes et déprimants. équivoque entre l'univers des bas-fonds et celui de l'élite sera exploité par la fiction et un bon nombre d'hommes de lettres brésiliens utiliseront les techniques du journalisme dans leurs œuvres littéraires.

aucoup d'entre eux, depuis le XIX ${ }^{e}$ siècle - comme José de Alencar, Machado de Assis, Aluísio Azevedo et Lima Barreto - ont écrit dans des journaux et ont inclus dans leur écriture les procédés de la presse quotidienne. Lima Barreto, par exemple, s'est engagé dans un reportage sur les excavations du Morro do Castelo à Rio de Janeiro où il a eu recours aux procédés narratifs du récit de fait divers. Quant à João do Rio, maître dans l'observation, il rend compte des mystères de la vie de la populace à une élite avide de la connaître. Et dans ces échanges entre le langage des faits divers et celui de la littérature, il y a plusieurs autres exemples d'Alcântara Machado à Oswald de Andrade, de Sylvio Floreal à Nelson Rodrigues, de Manuel Bandeira à Sérgio Porto. 

les titres démontrent de quelle façon les types de la pègre étaient privilégiés par sa plume : «Os Párias - uma noite no albergue noturno » («Les Parias - une nuit dans une auberge nocturne », 12/09/1921) et "O Inferno - duas horas na cadeia pública» («L'Enfer - deux heures à la prison publique », 04/10/1921). Ces textes reprennent, par le biais de la littérature, l'esthétique des bas-fonds qu'exploite de journal dans les faits divers.

Cette même confusion des genres choque la critique dans le cas de Os Condenados (Les Condamnés), roman d'Oswald de Andrade. Cette partie de sa Trilogia do Exilio (La Trilogie de l'Exil) apparaît pour la première fois en 1922, durant la Semaine d'Art Moderne. D'autres auteurs oubliés des compilations littéraires et boycottés par le modernisme paulista traitent des mêmes thèmes de manière explicite - ce qui a sans doute contribué à leur oubli. Mme Chrysanthème, pseudonyme de l'écrivain et journaliste Cecília Bandeira de Melo Rebelo de Vasconcelos publie Enervadas (1922), œuvre qui met en scène les bas-fonds et dont les personnages ont servi d'inspiration au caricaturiste J. Carlos pour ses « melindrosas " (cocottes) (Resende 1998, p. 19). Benjamin Costallat, auteur de livres populaires et de best-sellers du début du XXe siècle, alimente cet imaginaire dans lequel une femme dépendante à la cocaïne apparaît au premier plan. Elle est entourée de riches hommes avec lesquels elle passe ses nuits. Son personnage, Mademoiselle Cinema, est plein de manies françaises qu'il était à la mode d'imiter (Resende 1998, p. 21).

Les Paradis Artificiels de Charles Baudelaire ont certainement influencé cette production littéraire. La nouveauté constituée par la cocaïne a non seulement motivé les écrivains à tenter l'aventure de ce nouvel excitant, mais elle aurait également conduit une partie de la jeunesse au vice, suscitant ainsi l'inquiétude des journaux.

Plusieurs écrivains étaient aussi journalistes. Ils ont apporté dans les pages des journaux une écriture imprégnée de l'imaginaire des bas-fonds, influencée par Eugène Sue ${ }^{8}$ et reprise par Costallat dans ses Mistérios do Rio. Également inspiré par les «Mystères » de Londres et de New York, son récit a été publié en chroniques par le Jornal do Brasil et édité en livre pour la première fois en 1924. Travaillant comme rédacteur dans ce journal et dans la Gazeta de Notícias, il transfère les thèmes du fait divers dans ses fictions: les personnages stéréotypés et un parti pris moralisateur dominent dans sa prose, avec la prétention de faire un portrait fidèle de la réalité, comme l'explique l'auteur. La première chronique publiée s'intitule « No bairro da cocaína ». Le quartier de Glória, les cafés de Lapa et les « culs-de-sac pourris de la prostitution » (Costallat 1990, p. 25) offrent le cadre du crime. Comme les autres journalistes, Costallat rend compte des bas-fonds par une exposition minutieuse. Et on voit que même ses confrères n'échappent pas au terrible trafic :

Par l'action d'une série infinie d'intermédiaires, de revendeurs, la cocaïne est rapidement distribuée partout. Chauffeurs, " rapides ", garçons, prostituées, joueurs, et même vendeurs de légumes et de poissons, manucures, barbiers, dentistes, médecins, presque toutes les classes ont un représentant revendeur de drogue. Je connais même un journaliste qui, insatisfait de ce qui lui offrait la plume, était aussi revendeur de cocaïne. (Costallat 1990, p. 21)

50 Dans sa description d'un trafiquant qui traînait dans la "pissoire de la Glória ", on retrouve le personnage $d u$ « fripon carioca » :

C'est un mulâtre. Des bons vêtements, mais usés. Des vêtements achetés dans les friperies... 
Sous son chapeau de feutre, deux yeux brillent, étranges, fébriles, malades...

Des yeux de drogué. Des yeux de renifleur. (Costallat 1990, p. 22)

51 Les prostituées françaises, l'argot du crime et la "jeunesse dorée » (le terme est employé en français), complètent ce panorama du vice. À São Paulo, Hilário Tácito publiera un texte sur le même thème, sans pourtant faire référence à la consommation de drogue. Il dessine un portrait burlesque des bas-fonds de São Paulo, en faisant référence à un bordel de luxe qui a réellement existé et dont la propriétaire s'appelait Mme Pommery, laquelle donne son nom au livre. Comme Voltolino, il se rend compte du potentiel humoristique des inversions qui unissent la richesse à l'univers de l'underworld. Si les faits divers nous font comprendre les références qui construisent les normes sociales, l'humour des dessins et celui de la littérature démasque ce qui apparaît sous la plume du chroniqueur.

Madame Pommery (Tácito 1919[1998]), l'unique livre de l'ingénieur José Maria de Toledo Malta - le vrai nom de Hilário Tácito - présente les inversions entre les bas-fonds et l'élite de manière explicite, dans un style comique qui apparaît dès le nom de l'auteur, littéralement «Hilare Tacite ». Il fait une « critique bonhomme mais ferme de la société des propriétaires terriens et des hommes politiques de la civilisation du café ${ }^{\text {. Surtout en }}$ ce qui concerne leur vie bohème et leurs aventures extraconjugales. » (1919 [1998, p. 8]).

En dépit de son nom français, la propriétaire de la maison close, Madame Pommery, est en réalité une Espagnole, vendue par son père. Elle arrive au Brésil à bord du cargo «Bonne Chance» (en français, dans le texte). L'auteur raille l'habitude d'attribuer à n'importe quelle prostituée le qualificatif de "française », gage de séduction. Madame Pommery est un personnage réel, bien connu de la police de l'époque, mais Hilário Tácito en donne une version fictive. Dénonçant une ambiance excessivement francophone, l'auteur place l'élite et les bas-fonds dos à dos, tout en s'amusant des résultats de ces inversions. Ainsi, le débarquement de Madame Pommery à Santos est décrit comme celui d'une marchandise européenne parmi d'autres: "quinze tonneaux de vin de Bordeaux, des sardines, de la morue, deux mille volumes de Zola, du suif, quarante boîtes de champagne - et Mme Pommery » (1919 [1998, p. 44]).

Elle arrive pendant la " crise du café », c'est-à-dire, vers 1906, quand est signé l'Accord de Taubaté, qui assure à l'élite caféicultrice le pouvoir au détriment de la population. C'est à la même époque que la presse nationale se dote de rubriques exclusives pour les faits divers, et que São Paulo connaît une croissance intense et subite.

Dans le livre, l'histoire de São Paulo présente un événement marquant : l'ouverture d'un bordel luxueux, Paradis Retrouvé, de Madame Pommery, où le champagne français - signe évident de distinction - est servi. La consommation de drogue est interdite, car il s'agit d'une pension « de famille » - indication clairement ironique. Elle est fréquentée par tous les hommes de la haute société, de jeunes avocats, des journalistes et même par le chef de la police. Ainsi, la même société qui mène les campagnes moralisantes, comme dans $A$ Gazeta, se retrouve représentée dans la maison close.

Ce récit littéraire, comme les autres que nous avons cités, signale les progrès de références culturelles françaises, perçues comme les signes d'habitudes "civilisées »mais vues avec suspicion par les esprits plus critiques. L'ironie chez Madame Pommery ou dans les dessins de Voltolino se trouve justement dans la construction d'une relation ambiguë entre les représentations séduisantes des bas-fonds et la réalité brésilienne. Donner à l'actualité, prétendument vraie, des airs de roman français provoquait le rire et la moquerie des humoristes. Et, on peut affirmer, grâce aux exemples analysés, que le 
dessin humoristique apparait comme la meilleure expression d'une ironie qui passe par l'exagération du trait, l'échange des rôles, l'abaissement du noble et l'anoblissement de la foule.

57 C'est toujours dans la jeune presse à sensations brésilienne, et plus précisément dans les faits divers, qu'humoristes et écrivains modernistes trouvent leurs matériaux. Ces hommes des lettres rendent possible une représentation singulière des bas-fonds sous les tropiques, une «Belle Époque à la brésilienne » que Voltolino et ses contemporains ont très bien su adapter aux pages des journaux et des livres.

\section{BIBLIOGRAPHY}

ANDERSON, Benedict. Comunidades Imaginadas - reflexão sobre as origens e a difusão do nacionalismo. São Paulo: Cia. das Letras, 1983 [2008].

CHARLE, Christophe. Le Siècle de la Presse (1830-1939). Paris: Seuil, L'Univers Historique, 2004.

KALIFA, Dominique. L'Encre et Le Sang - récits de crimes et société à la Belle Époque. Paris: Fayard, 1995.

KALIFA, D. Les Bas fonds - histoire d'un imaginaire. Paris : Seuil, 2013.

MACHADO, Antônio de Alcântara. Brás, Bixiga e Barra Funda - notícias de São Paulo. SP: Imesp/Daesp. 1983. $1^{\text {a }}$ edição de 1927 [1982].

MEYER, Marlyse. Folhetim : uma história. São Paulo : Cia. das Letras, 1996.

MOTTA, Luiz Gonzaga (2002). "Teoria da Notícia: as relações entre o real e o simbólico", in: MOUILLARD, Maurice e PORTO, Sérgio Dayrell (orgs.), o Jornal - da forma ao sentido, 2 ed., Brasília: Ed. UNB (Coleção Comunicação, 2).

RESENDE, Beatriz. Cocaína. Rio de Janeiro: Casa da Palavra, 2006.

SEVCENKO, Nicolau. Orfeu extático na Metrópole - São Paulo, sociedade e cultura nos frementes anos 20. São Paulo: Companhia das Letras, 1992 [1998].

SHORE, Heather. "Undiscovered country": towards a history of the criminal "underworld", in Crimes and Misdemeanours, I, pp. 41-68, 2007.

SMYTHE, Ted Curtis. The Gilded Age Press (1865-1900). Westport: Praeger Publishers, 2003.

THERENTY, Marie-Ève; VAILLANT, Alain. 1836 : l'an 1 de l'ère médiatique: analyse littéraire et historique de la Presse de Girardin. Paris: Nouveau Monde, 2002.

Sources

Livres

BAUDELAIRE, Charles. Les Paradis Artificiels. Paris: Garnier-Flammarion, 2008.

COSTALLAT, Benjamim. Mistérios do Rio. Rio de Janeiro: DGSIC-SMCTE-RJ. Biblioteca Carioca (v. 14), organizado Afonso Carlos Marques dos Santos, 1990.

FLOREAL, Sylvio. Vícios, misérias e esplendores da Cidade de São Paulo. SP: Boitempo Editorial, 2002. 
FONSECA, Guido. Crimes, criminosos e criminalidade em São Paulo (1870-1950). São Paulo: Editora Resenha Tributária, 1988.

MOREYRA, Álvaro. "Cuidado!" (1923), in RESENDE, Beatriz. Cocaína. Rio de Janeiro: Casa da Palavra, 2006.

TÁCITO, Hilário. Madame Pommery. São Paulo: Editora Ática, 1919 [1998].

Journaux

SÃo PAULO

A Gazeta

O Estado de S. Paulo

Rio de Janeiro

Correio da Manhã

Gazeta de Notícias

Jornal do Brasil

Jornal do Comércio

\section{NOTES}

1. Voltolino, pseudonyme de Lemmo Lemmi, était un des grands noms brésiliens qui se consacrait à l'art de Daumier. Il était extrêmement populaire dans la São Paulo des années 20 aussi bien pour les dessins d'hommes politiques que pour ses textes de journaliste. Habitué de la zone bohémienne et du centre ville, il était paulista d'origine italienne, né en 1884 . De 1886 à 1904, il a habité à Pise, en Italie, où il a étudié le dessin. De retour à São Paulo, il publie des dessins dans plusieurs organes comme les revues Cara Dura, Il Grillo di Flora, O Malho, O Pirralho (avec le moderniste Oswald de Andrade), A Vespa et beaucoup d'autres. Il a été le créateur de personnages emblématiques de la presse nationale comme Juó Bananére (João Bananeiro/Jean Bananière), une fusion du Brésilien avec l'Italien, autrement dit un paulista qui a un discours italianisé dans une République... Bananière.

2. "O Vício Chic - Mais uma nobre campanha contra a cocaína avassaladora", por Voltolino.

3. Ensemble des trois principales rues du centre-ville de São Paulo: Rua Direita, Rua XV de Novembro e Rua São Bento.

4. KHOURY, Y. A. As greves de 1917 em São Paulo e o processo de organização proletária. São Paulo: Cortez, 1981; BIONDI, Luigi. “A greve geral de 1917 em São Paulo e a imigração italiana: novas perspectivas” in Campinas: Cadernos AEL, v.15, n. 27, 2009, pp. 262-308.

5. Autour du suicide par overdose, on peut lire les nouvelles : «Vontade de Morrer» (« Envie de mourir » O Estado de S. Paulo, 23/01/1912) (alcool); «Intoxicação por cocaína » («Intoxication par la cocaïne » O Estado de S. Paulo, 07/02/1917); « Tentativa de suicídio » (« Tentative de suicide » 0 Estado de S. Paulo, 16/01/1917).

6. «A cocaína avassalou São Paulo ».

7. Publié en avril 1905 au journal Correio da Manhã.

8. Le livre Mystères de Paris, d'Eugène Sue, est la première et principale référence, donc la parution en France a été publiée en feuilleton dans le Journal des Débats entre 1842 et 1843. Toutefois, Costallat nie sa filiation à cet auteur ou même à Paul Féval, auteur des Mystères de Londres. 
9. La « civilisation du café » est une expression qui désigne la société paulista entre 1860 et 1930 , dont l'économie s'est structurée fortement dans les plantations et qui repose sur la monoculture du café, le travail d'esclaves et une production tournée à l'exportation. Le travail des esclaves laisse place après 1888, date de la loi d'Abolition, à des immigrants, la plupart italiens.

\section{AUTHOR}

\section{VALÉRIA GUIMARÃES}

Valéria Guimarães

Professeur d'Histoire Culturelle, Universidade Estadual Paulista (UNESP), São Paulo, Brésil FAPESP 